\title{
Alopecia syphilitica diffusa
}

\author{
Alexander Zink ${ }^{1,2} \cdot$ Katharina Kaliebe ${ }^{1,2} \cdot$ Christoph D. Spinner ${ }^{2,3}$
}

Received: 17 February 2015 / Accepted: 21 April 2015 / Published online: 1 May 2015

(C) Springer-Verlag Berlin Heidelberg 2015

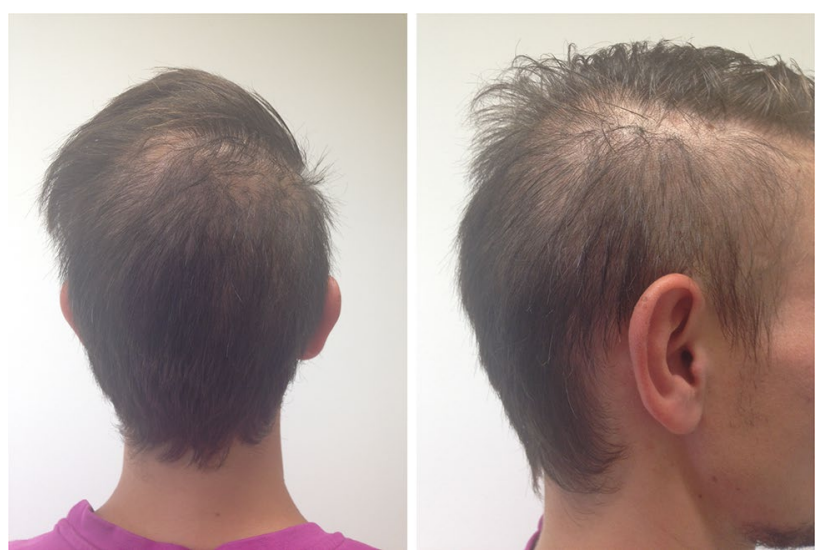

A 27-year-old HIV-positive man (CDC stadium A2) on stable antiretroviral therapy with tenofovir disoproxil fumarate/emtricitabine/rilpivirine (TDF/FTC/RPV) (CD4 cell count $960 / \mu$ l, HIV RNA not detectable) presented with clinical findings of a progressive hair loss on his scalp within only a few weeks. On request, a painless ulceration on the palate 6 weeks prior to actual presentation after unprotected oral intercourse with another man has been reported. On physical examination, a $1 \mathrm{~cm}$ in diameter

Alexander Zink

zink@1rz.tum.de

1 Department of Dermatology and Allergy, University Hospital Klinikum rechts der Isar, Technische Universität München, Biedersteiner Str. 29, 80802 Munich, Germany

2 Interdisciplinary HIV Centre (IZAR) at University Hospital Klinikum rechts der Isar, Technische Universität München, Munich, Germany

3 Department of Medicine II, University Hospital Klinikum rechts der Isar, Technische Universität München, Munich, Germany large ulceration was observed on the mucosa of the hard palatine, as well as cervical lymphadenopathy. Laboratory evaluation revealed a reactive Treponema pallidum particle agglutination assay (TPPA) and a Venereal Disease Research Laboratory (VDRL) test of 1:256 with known negative TPPA testing 5 months prior to actual presentation. Diagnosis of secondary syphilis with alopecia syphilitica diffusa, lymphadenopathy and persisting hard chancre was made. A treatment with a single gluteal, intramuscular injection of benzylpenicillin 2.4 million units [1] following $60 \mathrm{mg}$ prednisolone per os as prophylaxis against JarischHerxheimer reaction led to complete resolved symptoms. Alopecia syphilitica is a rare and rather uncommon manifestation of secondary syphilis occurring in only about $5 \%$ of syphilis patients [2]. It can be the only clinical symptom of syphilis infection and mimic alopecia areata in clinical course or on histopathological findings [3]. Syphilitic hair loss, suggested to be caused by the direct infection of hair follicles by Treponema pallidum [4], is non-inflammatory and noncicatricial and typically presents in a diffuse or in a moth-eaten pattern with multiple patches of non-scarring alopecia, or in a combination of both $[5,6]$.

Conflict of interest On behalf of all authors, the corresponding author states that there is no conflict of interest.

\section{References}

1. Janier M, Hegyi V, Dupin N, Unemo M, Tiplica GS, Potočnik M, French P, Patel R. 2014 European guideline on the management of syphilis. J Eur Acad Dermatol Venereol. 2014;28:1581-93.

2. Bi MY, Cohen PR, Robinson FW, Gray JM. Alopecia syphilitica-report of a patient with secondary syphilis presenting as moth-eaten alopecia and a review of its common mimickers. Dermatol Online J. 2009;15:6. 
3. Jordaan HF, Louw M. The moth-eaten alopecia of secondary syphilis. A histopathological study of 12 patients. Am J Dermatopathol. 1995;17:158-62.

4. Nam-Cha SH, Guhl G, Fernández-Peña P, Fraga J. Alopecia syphilitica with detection of Treponema pallidum in the hair follicle. J Cutan Pathol. 2007;34(Suppl 1):37-40.

5. Lee JY, Hsu ML. Alopecia syphilitica, a simulator of alopecia areata: histopathology and differential diagnosis. J Cutan Pathol. 1991;18:87-92.
6. SpornraftRagaller P, Boashie U, Stephan V, Schmitt J. Analysis of risk factors for late presentation in a cohort of HIVinfected patients in Dresden: positive serology for syphilis in MSM is a determinant for earlier HIV diagnosis. Infection. 2013;41:1145-55. 